\title{
Measuring the Head Circumference on MRI in Children: an Interrater Study
}

\author{
Alexander Rau ${ }^{1}$ (D) $\cdot$ Theo Demerath $^{1} \cdot$ Nico Kremers $^{1} \cdot$ Matthias Eckenweiler ${ }^{2}$ Rieka von der Warth ${ }^{3}$. \\ Horst Urbach ${ }^{1}$
}

Received: 19 February 2021 / Accepted: 11 April 2021 / Published online: 21 May 2021

(c) The Author(s) 2021

\begin{abstract}
Purpose The head circumference is typically used as a surrogate parameter for the development of the central nervous system and intracranial structures and is an important clinical parameter in neuropediatrics. As magnetic resonance images (MRI) can be freely zoomed, visual analysis of the head size often relies on impressions, such as the craniofacial ratio or a simplified gyral pattern. Aim of this study was to validate an MRI-based method to measure the head circumference. Methods Head circumferences of 85 children (41 microcephalies, 22 macrocephalies and 22 normal controls; 47 male, mean age $3.22 \pm 2.45$ years, range $0.19-10.42$ years) were retrospectively measured using sagittal 3D-T1w (MPRAGE) data sets. Three readers independently placed an ovoid region of interest in an axial plane starting from the supraorbital bulge and covering the largest supra-auricular head circumference. Clinical measurements of the head circumference taken within an acceptable period served for comparative purposes. Reliability was assessed by calculating the total error of measurement (TEM) and the intraclass correlation coefficient (ICC).

Results A close correlation was found between MRI-based and clinical measurements. The interrater reliability was excellent (ICC 0.985, 95\% confidence interval 0.952-0.993). Absolute TEM ranged from 0.47-0.75, resulting in relative TEM ranging from 1.0-1.6\%. Thus, TEMs were classified as acceptable. The mean accuracy of MRI-based measurements was high at 0.94 .

Conclusion The head circumference can be reliably determined with a simple measurement on 3D sequences using multiplanar reformations. This approach may help to diagnose microcephaly and macrocephaly, especially when the head circumference is not reported by the referring physician.
\end{abstract}

Keywords Microcephaly $\cdot$ Megalocephaly $\cdot$ Cephalometry $\cdot$ Macrocephaly $\cdot$ Neuropediatrics

Availability of data On request.

Code availability On request.

Consent to participate Waived by the institutional review board.

Consent for publication Not applicable.

Alexander Rau

alexander.rau@uniklinik-freiburg.de

1 Dept. of Neuroradiology, University Medical Center Freiburg, Freiburg, Germany

2 Dept. of Neuropediatrics and Muscle Disorders, University Medical Center Freiburg, Freiburg, Germany

3 Institute of Medical Biometry and Statistics, Section of Health Care Research and Rehabilitation Research, Medical Center - University of Freiburg, Faculty of Medicine, University of Freiburg, Freiburg, Germany

\section{Abbreviations}

$\mathrm{HC} \quad$ Head circumference

ICC Intraclass correlation coefficient

ROI Region of interest

TEM Technical errors of measurements

\section{Key Points}

- Head circumference was measured based on 3D T1weighted magnetic resonance imaging (MRI) datasets.

- Retrospective interrater study showed close correlation of clinical and radiological measurements.

- Radiologically measured head circumference showed high accuracy and reliability. 


\section{Introduction}

The head circumference (HC) is a validated parameter of a pediatric examination. It is easily measured and used as surrogate parameter for the development of the central nervous system and intracranial structures [1].

The circumference of the skull starting from the supraorbital bulge is determined using a measuring tape [2, 3]. Agespecific percentile curves are derived from large data collec- tions [3]. Deviations of more than two standard deviations are considered as abnormal: $\mathrm{HC}$ values below the $3 \mathrm{rd}$ percentile define microcephaly and above the 97th macrocephaly $[4,5]$.

Microcephaly affects approximately 1.6/1000 live births and is often associated with a developmental delay [6]. Other common comorbidities include epilepsy, cerebral palsy, and mental retardation. The causes include genetic
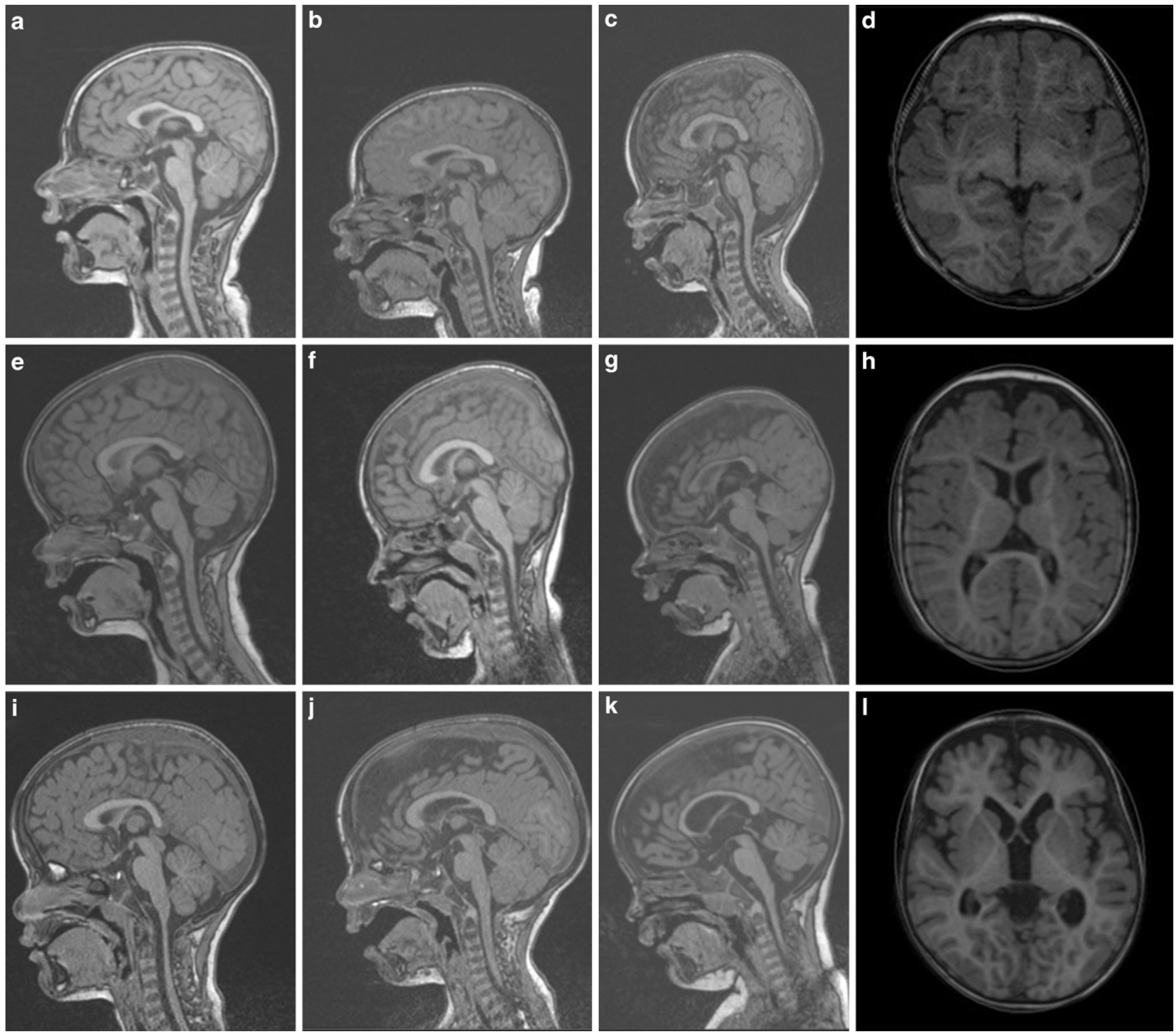

Fig. 1 Midsagittal reformats of 3D T1-weighted MPRAGE (Magnetization Prepared - RApid Gradient Echo) sequences with FOV height of $25 \mathrm{~cm}$. As children have different ages (10 months to 3 years) and FOVs are adapted to the head sizes as depicted, it is difficult to assess which children are microcephalic, have a normal head size or are macrocephalic. Microcephaly: a male, 3 years, $47 \mathrm{~cm} \mathrm{HC}(0.7 \mathrm{~cm}<3 \mathrm{rd}$ percentile), b male, 4 years, $47 \mathrm{~cm} \mathrm{HC}\left(1.2 \mathrm{~cm}\right.$ below the $3 \mathrm{rd}$ percentile), c male, 15 months, $43 \mathrm{~cm} \mathrm{HC}\left(1 \mathrm{~cm}<3 \mathrm{rd}\right.$ percentile). Normal HC: e female, $3 \frac{10}{12}$ years, $51.5 \mathrm{~cm} \mathrm{HC}$ ( $92 \mathrm{nd}$ percentile), f male, $3 \frac{4}{12}$ years, $50 \mathrm{~cm} \mathrm{HC}$ (24th percentile), g male, 10 months, $45 \mathrm{~cm}$ HC (33rd percentile). Macrocephaly: i male, $3 \frac{10}{12}$ years, $53 \mathrm{~cm} \mathrm{HC}(0.5 \mathrm{~cm}>97$ th percentile $), \mathbf{j}$ male, $3 \frac{4}{12}$ years, $56.2 \mathrm{~cm} \mathrm{HC}(2.8 \mathrm{~cm}>99$ th percentile $), \mathbf{k}$ female, $24 \mathrm{months}, 51 \mathrm{~cm} \mathrm{HC}$ $(0.5 \mathrm{~cm}>97$ th percentile). By measuring the head circumference, which is exemplary shown in the right-hand column, a child's head size can be easily classified: d (case c) clinical HC $43 \mathrm{~cm}$, MRI-based HC $435 \mathrm{~mm}$, h (case g) clinical HC $45 \mathrm{~cm}$, MRI-based HC $440 \mathrm{~mm}, \mathbf{l}$ (case k) clinical HC $51 \mathrm{~cm}$, MRI-based HC $505 \mathrm{~mm}$ 

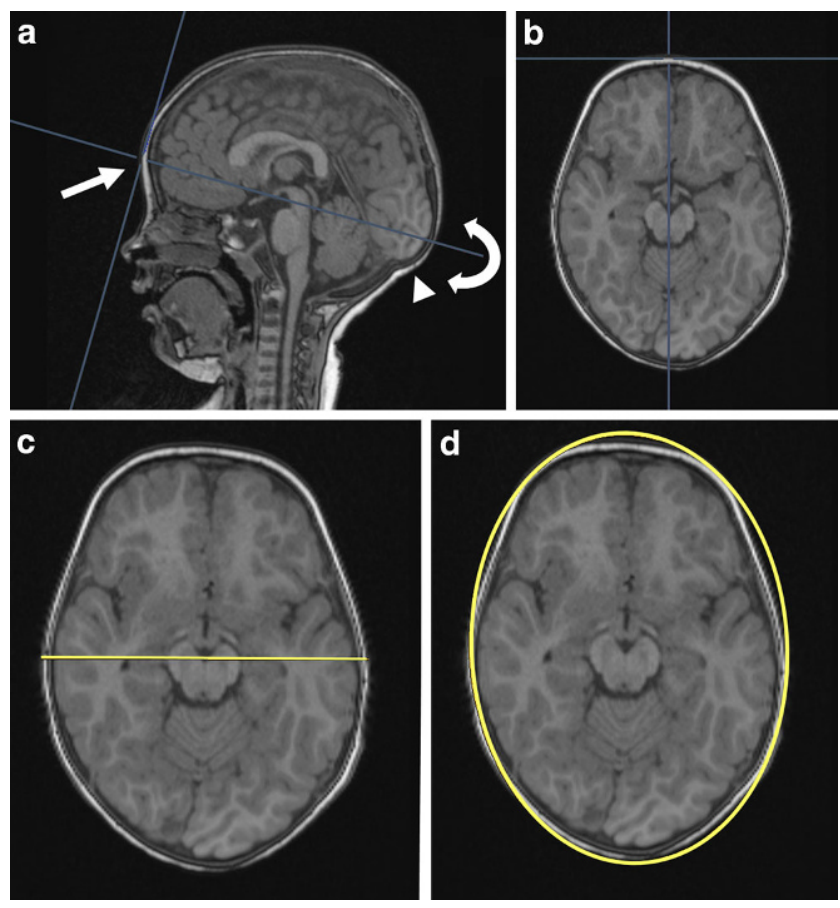

Fig. 2 A 2-year-old girl with microcephaly (clinical head circumference $45 \mathrm{~cm} /<1$ st percentile) a Midsagittal MPRAGE (Magnetization Prepared - RApid Gradient Echo) 3D-reformat with a nearly normal craniofacial ratio. For MRI-based head circumference estimation identify the supraorbital bulge (white arrow). Then adapt the axial plane in the 3D-reformation (depicted by the white curved arrow) until the largest supra-auricular head circumference is achieved (as depicted in b). The inion (white arrowhead) can be used as a landmark. c, $\mathbf{d}$ An ovoid ROI is created in axial reformat by first identifying the lateral expansion (yellow line in c) and then the anteroposterior expansion (d). MRI-based head circumference is $45.1 \mathrm{~cm}$

syndromes, environmental toxins, infectious diseases and structural brain disorders [7].

Macrocephaly affects up to $5 \%$ of pediatric patients and is often caused by a disturbance in the cerebrospinal fluid (CSF) circulation [8] while genetic syndromes are associated with macrocephaly, too [9].

Currently, there are only limited data on the correlation between $\mathrm{HC}$ and imaging features of microcephaly/macrocephaly and associated cerebral malformations [10-14].

If a pathological $\mathrm{HC}$ is documented, magnetic resonance imaging (MRI) is recommended to further evaluate the intracranial structures and investigate possible underlying pathologies [15].

Since the HC is not always documented by referring physicians, radiologists might feel the need to measure the HC using MRI; however, it is difficult to assess the head size on MRI as the head size is age-dependent and the field of view (FOV) on MRI is typically adapted to the head size in order to increase the spatial resolution, but different FOV are not apparent. Positioning of the head within the head coil may be variable, and different reformations of the increasingly acquired 3D sequences make the reader's interpretation difficult (see Fig. 1 for illustration). Thus, assessment of the head size is often limited to the craniofacial ratio while objective measurements are lacking. The craniofacial ratio is defined as the ratio of the area of the intracranial structures to the area of the face on midsagittal reconstructions. While only subjective values can be evaluated and no normal values exist, it is known to be large at birth and decreases with increasing age [10].

Knowledge of the $\mathrm{HC}$ is essential and might facilitate evaluation of pediatric brain imaging; however, repeated clinical tape measurement is not feasible during clinical routine, emphasizing the need for a simple MRI-based method to measure the HC.

Thus, the purpose of this retrospective study was to develop a simple MRI-based measurement of the head circumference and to validate it by comparing MRI-based with clinical measurements in pediatric patients.

\section{Methods}

This trial was carried out according to the guidelines for reporting reliability and agreement studies [16]. This study was performed in line with the principles of the Declaration of Helsinki. Approval was granted by the local ethics committee, informed written consent was waived.

\section{Assessment of HC}

We retrospectively included patients who had been admitted to the department of pediatrics and underwent cranial MRI with a sagittal 3D MPRAGE (Magnetization Prepared RApid Gradient Echo) sequence using a $1.5 \mathrm{~T}$ Siemens Avanto with a 12-channel head coil and a $3 \mathrm{~T}$ Siemens Trio scanner (Siemens Healthcare, Erlangen, Germany) with a 32-channel head coil, respectively. Patients had to be younger than 18 years and a clinically documented HC within an acceptable period before or after the MRI study measured by an experienced clinician was necessary. In order to take the age-dependent dynamics of head growth into account, the time intervals between imaging and clinical measurement were limited. Accordingly, for younger patients with relatively faster growth, a shorter time period was chosen. This acceptable period was defined as 3 months for children older than 6 years, as 1 month for children aged 2-6 years and as 1 week for children younger than 2 years. To ensure that a sufficient number of pathological $\mathrm{HC}$ were included a selected sampling approach was chosen, initially identifying 20 children with microcephaly and 20 with macrocephaly by a report query. A total of 45 more 
patients were then included by means of a backward case tracking based on MRI examinations and existing clinical data.

The three readers $(6,4$, and 3 years of experience in clinical neuroradiology) independently reviewed the MR images using the in-house Picture Archiving and Communication System (PACS) (IMPAX EE R20 VXII SU4, Agfa HealthCare N.V., Mortsel, Belgium) with application of the $3 \mathrm{D}$ reformations plug-in, being blinded for patient age, gender and clinical data. An ovoid region of interest (ROI) was placed in an axial plane starting from the supraorbital bulge and covering the largest supra-auricular HC (Fig. 2). If the head shape was asymmetric, raters were advised to place the ROI as accurate as possible with the aim of reproducing the outer circumference as accurately as possible. Individual values were transformed into percentiles [3]. Following the World Health Organization (WHO) 2016 guidelines, the 3 rd percentile was chosen as a threshold for a pathologically small head circumference $[4,5]$ and the 97 th percentile was used for macrocephaly.

All readings were independently performed in summer 2020 at the department of neuroradiology. For training purposes, readers were provided with the clinical HC measurement once the MRI-based measurement was performed in the first nine cases.

\section{Statistical Analysis}

Interrater reliability was assessed using the intraclass correlation coefficient (ICC) based on a two-way random effects model [17]. Also, absolute and relative technical errors of measurements (TEM) (variation of measurements performed by different anthropometrists in the same group of persons) were calculated as proposed by Perini et al. [18]. Accuracy was calculated according to Baratloo et al. [19].

A comparison between the MRI-based and clinical measurements using a measuring tape was done on a descriptive level, testing for linearity using a scatterplot. All analyses were performed using SPSS Statistics 26 (IBM Corporation, Armonk, NY, USA) and Microsoft Excel 2010 (Microsoft Corporation, Redmond, WA, USA).

\section{Results}

\section{Sample}

This study included 85 children, 47 (55.3\%) male and $38(44.7 \%)$ female. Mean age at the time of clinical measurement was 3.18 years (standard deviation [SD] 2.45 years, range $0.21-10.77$ years) and 3.22 years (SD
Table 1 Head circumference values (in $\mathrm{cm}$ ) determined by clinical tape measurement and MRI-based measurements

\begin{tabular}{llll}
\hline & Mean & SD & Range \\
\hline Tape measurement & 47.92 & 4.92 & $36.5-56.6$ \\
Reader 1 & 47.92 & 4.86 & $36.4-56.6$ \\
Reader 2 & 48.35 & 4.78 & $36.7-56.9$ \\
Reader 3 & 47.55 & 4.78 & $36.7-56.5$ \\
\hline
\end{tabular}

SD Standard deviation

Table 2 Technical error of MRI-based measurements between readers

\begin{tabular}{llll}
\hline Reader & $\begin{array}{l}\text { Absolute } \\
\text { TEM }\end{array}$ & Relative TEM $(\%)$ & Classification \\
\hline $1-2$ & 0.52 & 1.1 & Acceptable \\
$1-3$ & 0.47 & 1.0 & Acceptable \\
$2-3$ & 0.75 & 1.6 & Acceptable $^{\mathrm{a}}$ \\
\hline
\end{tabular}

Classification based on Perini et al. [18]

TEM technical errors of measurements

${ }^{a}$ Acceptable for readers at beginner level

2.45 years, range $0.19-10.42$ years) at the time of MRI based measurement, respectively. Based on clinical tape measurements, 41 patients were microcephalic (48.2\%; of whom 37 were diagnosed with severe microcephaly $\leq 1$ st percentile), 22 were macrocephalic $(25.9 \%$; 16 with severe macrocephaly $\geq 99$ th percentile) and $22(25.9 \%)$ had a normal HC. The HC ranged from $36-56 \mathrm{~cm}$ in both clinical tape measurement and MRI measurement. See Table 1 for details.

\section{Interrater Reliability}

Interrater agreement was excellent with an ICC of 0.985 (95\% confidence interval 0.952-0.993). Absolute TEM ranged from $0.47-0.75$, resulting in relative TEM ranging from 1.0-1.6\%. Thus, TEMs were classified as acceptable.

The TEM are shown in Table 2, classification was based on that of Perini et al. [18].

\section{Accuracy of MRI-based Measurement}

Table 3 shows the accuracy of the MRI-based measurement, comparing it to the clinical tape measurement.

Rater 1 correctly identified 57 of 63 pathological HCs and 20 of 22 normal HCs. Rater 2 correctly assessed 54 pathological and 20 normal $\mathrm{HCs}$ and rater 3 identified 55 pathological and 18 normal HCs. Rater 2 missed 5 cases of severe microcephaly and rater 3 cases of severe macrocephaly as normal $\mathrm{HC}$, respectively. In those falsely classified cases, the MRI-based percentiles determined were close to the pathologic values. One case (male, 42 months old, clinical measurement 4 weeks prior to imaging) with a clinical measurement of $54 \mathrm{~cm}$ referring to the 99 th percentile was missed by all raters $(51.5 \mathrm{~cm} / 69$ th percentile, 
Table 3 Agreement between individual MRI-based ratings and tape-based measurement of head circumference

\begin{tabular}{|c|c|c|c|c|c|c|c|c|c|c|c|c|c|}
\hline & & \multicolumn{3}{|l|}{ Rater 1} & \multicolumn{3}{|c|}{ Rater 2} & \multicolumn{3}{|c|}{ Rater 3} & \multicolumn{3}{|c|}{ MRI-based mean } \\
\hline & & Micro & Norm & Macro & Micro & Norm & Macro & Micro & Norm & Macro & $\overline{\text { Micro }}$ & Norm & Macro \\
\hline \multirow{3}{*}{$\begin{array}{l}\text { Tape mea- } \\
\text { surement }\end{array}$} & Micro & 38 & 3 & 0 & 35 & 6 & 0 & 41 & 0 & 0 & 41 & 0 & 0 \\
\hline & Norm & 1 & 20 & 1 & 0 & 20 & 2 & 3 & 18 & 1 & 0 & 20 & 2 \\
\hline & Macro & 0 & 3 & 19 & 0 & 3 & 19 & 0 & 8 & 14 & 0 & 3 & 19 \\
\hline
\end{tabular}

The count of the respective allocations are indicated.

Micro microcephalic, Norm normocephalic, Macro macrocephalic

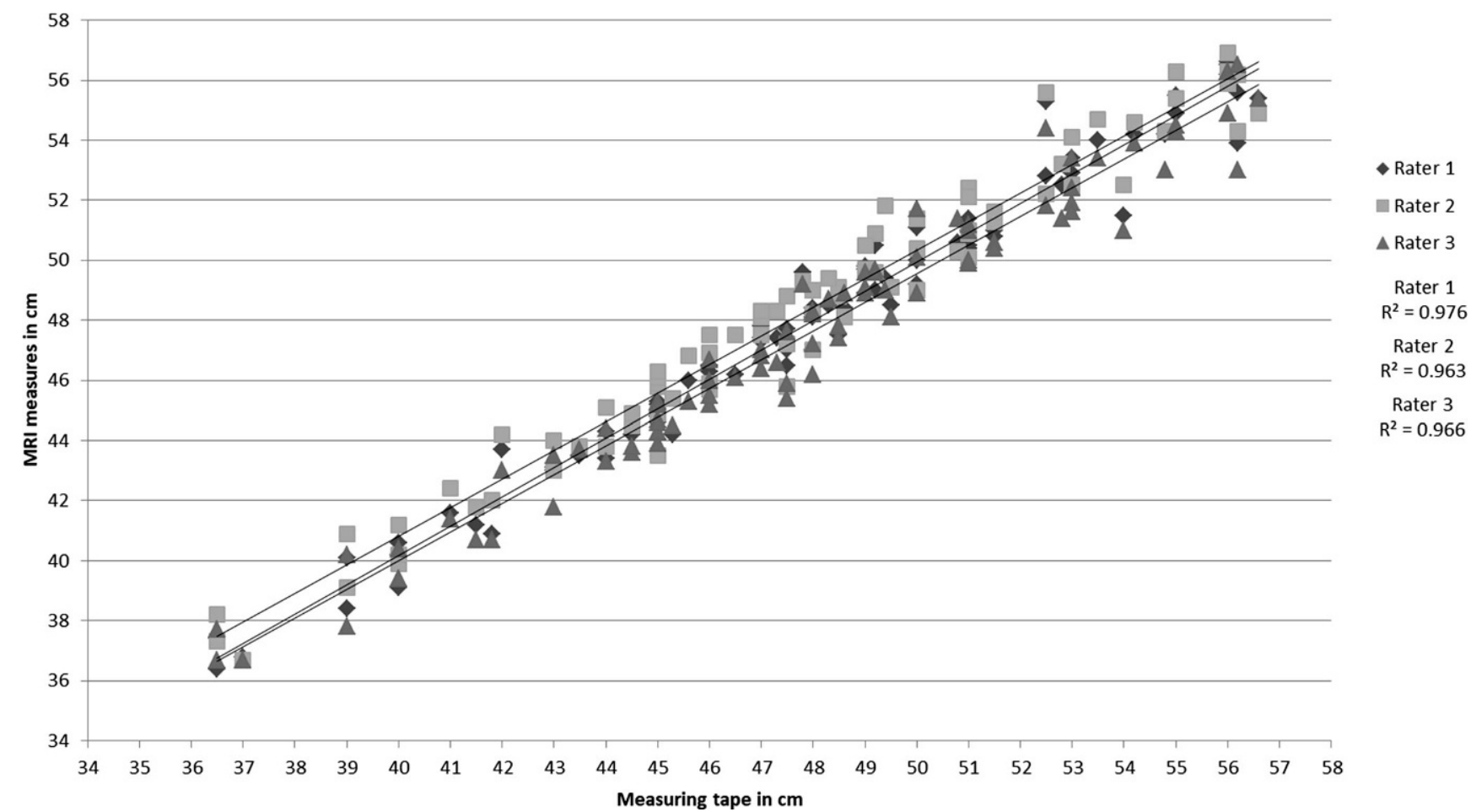

Fig. 3 Correlation of absolute clinical tape-based ( $\mathrm{X}$ axis; in $\mathrm{cm}$ ) and MRI-based (Y axis; in $\mathrm{cm}$ ) HC measurements

$52.5 \mathrm{~cm} / 90$ th percentile and $51.0 \mathrm{~cm} / 54$ th percentile). Another case was also falsely classified as normal by all raters: male, 10 years old, clinical measurement on the day of MRI with clinically $56.6 \mathrm{~cm} \mathrm{HC}$ referring to the 98th percentile and MRI-based $55.4 \mathrm{~cm}, 54.9 \mathrm{~cm}$ and $55.4 \mathrm{~cm}$.

Overall, the sensitivity of the MRI-based measurements was 0.97 , specificity 0.87 , and accuracy 0.94 .

Testing for linearity using a scatter plot, the regression models showed strong relationships between clinical measurements of MRI measurements. The linear correlation between clinical and MRI-based measurements is shown in Fig. 3.

\section{Discussion}

We evaluated an MRI-based HC measurement method compared it to tape measurement, being validated on both nor- mal and pathological HC. We present a simple, MRI-based algorithm to measure the head circumference (HC) and prove its accuracy by comparing MRI-based and tape measurements typically performed but often not reported by the referring physicians. The measurement requires acquisition of an ideally isotropic 3D sequence. Axial reformats are generated, covering the supraorbital bulge, for identification of the largest head circumference (Figs. 1 and 2).

Approaches on image-based HC estimation have so far been poorly validated. For instance, Smith et al. developed an automated, CT-based approach in which they derived the HC from a bone segmentation [11], while Vorperian et al. evaluated image-based measurements using two orthogonal lines at a level comparable to the clinical measurement to estimate HC [12]. In this latter approach, atypical head shapes were not taken into account, which might be disadvantageous, e.g. in the occurrence of craniosynostosis. Previous studies used clinically assessed HC in children 
with microcephaly and adults, which were correlated with intracranial volumes without radiologically verifying the clinical measurement $[12,14]$. Another study approximated pathological head sizes by examining sagittal 2D images in microcephaly patients [10]. Without clinical information regarding the $\mathrm{HC}$, images were evaluated concerning the craniofacial ratio and the microcephaly was classified into three severity levels using age-matched controls.

It is only in the last decade that $3 \mathrm{D}$ sequences have become widely available. Our method was performed using a commercially available PACS and 3D reformation plug-in in one-Vendor (IMPAX EE R20 VXII SU4, Agfa HealthCare N.V.), and in our opinion appears to be applicable in any routine clinical PACS environment without dedicated postprocessing tools.

Although rater 1 missed 3 microcephaly and 3 macrocephaly patients, rater 2 falsely classified 6 and 3, respectively, and rater 3 rated 8 macrocephalies as normal $\mathrm{HC}$, the accuracy was high. Thus, we would consider the MRI-based measurement reliable and fast (postprocessing time $<1 \mathrm{~min}$ ). A high interrater agreement also supports this conclusion [16]. More than the ICC, the TEM is a central parameter in anthropometric measurements, as the ICC does not account for bias in measurement [20]. Thus, results of the TEM show a more differentiated picture. While the TEMs of readers 1-2 and readers 1-3 were acceptable, the relative TEM of readers $2-3$ only reached $1.6 \%$. According to the current literature, trained readers should reach a relative TEM of $1.5 \%$ and below $[18,21]$. Readers in this study were trained neuroradiologists; however, as this study assessed a new measurement for the assessment of the HC, we would still consider all TEMs of acceptable quality. Accordingly, it can be assumed that the MRI-based HC measurement yields approximately reproducible and reliable $\mathrm{HC}$ values as the clinical tape measurement [22]. With respect to the missed pathological $\mathrm{HC}$, it should be mentioned that these were borderline percentiles.

Compared to previous studies, our study has the strength that we included a high number of pathological HC cases.

However, this study shows some limitations including the number of patients and the time period between clinical HC measurement and imaging. The retrospective design did not allow a recheck of the clinical measurements, and thus incorrect clinical measurements may occur in the data. The age range was limited to younger patients since $\mathrm{HC}$ measurement are not commonly acquired in adolescence. Additionally, due to the monocentric design an inherent risk of bias appears. The fact that the tape measurement $\mathrm{HC}$ values were revealed to the raters after the first nine measurements had no relevant effect on the results, since the MRI-based measurements were not allowed to be changed.

\section{Conclusion}

This study implies that the MRI-based measurement of the head circumference provides a reliable approximation of the established clinically measured head circumference. The increasing availability of $3 \mathrm{D}$ datasets presumably allows the simple reproduction of clinical measurements of the outer circumference of the head. Thus, this method of HC measurement can be very useful in both clinical and research settings.

Funding The authors state that this work has not received any funding.

Funding Open Access funding enabled and organized by Projekt DEAL.

\section{Declarations}

Conflict of interest The authors report no conflicts of interest. The authors of this manuscript declare no relationships with any companies, whose products or services may be related to the subject matter of the article.

Ethical standards All procedures performed in studies involving human participants or on human tissue were in accordance with the ethical standards of the institutional and/or national research committee and with the 1975 Helsinki declaration and its later amendments or comparable ethical standards. Informed consent was waived due to the retrospective nature of the study. Institutional review board approval was obtained.

Open Access This article is licensed under a Creative Commons Attribution 4.0 International License, which permits use, sharing, adaptation, distribution and reproduction in any medium or format, as long as you give appropriate credit to the original author(s) and the source, provide a link to the Creative Commons licence, and indicate if changes were made. The images or other third party material in this article are included in the article's Creative Commons licence, unless indicated otherwise in a credit line to the material. If material is not included in the article's Creative Commons licence and your intended use is not permitted by statutory regulation or exceeds the permitted use, you will need to obtain permission directly from the copyright holder. To view a copy of this licence, visit http://creativecommons.org/licenses/by/4. $0 /$.

\section{References}

1. Harris SR. Measuring head circumference: Update on infant microcephaly. Can Fam Physician. 2015;61:680-4.

2. Nellhaus G. Head circumference from birth to eighteen years. Practical composite international and interracial graphs. Pediatrics. 1968;41:106-14.

3. Schienkiewitz A, Schaffrath Rosario A, Dortschy R, Ellert U, Neuhauser H. German head circumference references for infants, children and adolescents in comparison with currently used national and international references. Acta Paediatr. 2011;100:e2833. Erratum in: Acta Paediatr. 2011; 100:1399-400.

4. World Health Organization. Screening, assessment and management of neonates and infants with complications associated with Zika virus exposure in utero. Rapid Advice Guideline. 2016. WHO/ZIKV/MOC/16.3/Rev3. Accessed 26 June 2020. 
5. Kaindl AM, Chaoui R, Hahn G, Hennermann J, Horn D, LandwehrKenzel S, Moog U, Niller HH, von Bernuth H, Rosenbaum T, Thomale UW, von der Hagen M. S2k-Leitlinie Klassifikation und Diagnostik der Mikrozephalie. AWMF-Leitlinie. 2019. www. awmf.org/leitlinien. Accessed 26 June 2020.

6. Baltzer J, Friese K, Graf M, Wolff F, editors. Praxis der Gynäkologie und Geburtshilfe. Stuttgart: Thieme; 2006.

7. Passemard S, Kaindl AM, Verloes A. Microcephaly. Handb Clin Neurol. 2013;111:129-41.

8. Tan AP, Mankad K, Gonçalves FG, Talenti G, Alexia E. Macrocephaly: Solving the Diagnostic Dilemma. Top Magn Reson Imaging. 2018;27:197-217.

9. Pirozzi F, Nelson B, Mirzaa G. From microcephaly to megalencephaly: determinants of brain size. Dialogues Clin Neurosci. 2018;20:267-82.

10. Adachi Y, Poduri A, Kawaguch A, Yoon G, Salih MA, Yamashita F, Walsh CA, Barkovich AJ. Congenital microcephaly with a simplified gyral pattern: associated findings and their significance. AJNR Am J Neuroradiol. 2011;32:1123-9.

11. Smith K, Politte D, Reiker G, Nolan TS, Hildebolt C, Mattson C, Tucker D, Prior F, Turovets S, Larson-Prior LJ. Automated measurement of skull circumference, cranial index, and braincase volume from pediatric computed tomography. Annu Int Conf IEEE Eng Med Biol Soc. 2013;2013:3977-80.

12. Vorperian HK, Durtschi RB, Wang S, Chung MK, Ziegert AJ, Gentry LR. Estimating head circumference from pediatric imaging studies an improved method. Acad Radiol. 2007;14:1102-7.

13. Cheong JL, Hunt RW, Anderson PJ, Howard K, Thompson DK, Wang HX, Bear MJ, Inder TE, Doyle LW. Head growth in preterm infants: correlation with magnetic resonance imaging and neurodevelopmental outcome. Pediatrics. 2008;121:e1534-40.

14. Hshieh TT, Fox ML, Kosar CM, Cavallari M, Guttmann CR, Alsop D, Marcantonio ER, Schmitt EM, Jones RN, Inouye SK. Head circumference as a useful surrogate for intracranial volume in older adults. Int Psychogeriatr. 2016;28:157-62.

15. Hanzlik E, Gigante J. Microcephaly. Children (Basel). 2017;4:47

16. Kottner J, Audigé L, Brorson S, Donner A, Gajewski BJ, Hróbjartsson A, Roberts C, Shoukri M, Streiner DL. Guidelines for Reporting Reliability and Agreement Studies (GRRAS) were proposed. J Clin Epidemiol. 2011;64:96-106.

17. Wirtz M, Caspar F. Beurteilerübereinstimmung und Beurteilerreliabilität. [Inter-rater agreement and inter-rater reliability]. Göttingen: Hogrefe; 2002.

18. Perini TA, De Oliveira GL, Ornellas JDS, De Oliveira FP. Technical error of measurement in anthropometry. Rev Bras Med Esporte. 2005;11:81-5.

19. Baratloo A, Hosseini M, Negida A, El Ashal G. Part 1: Simple Definition and Calculation of Accuracy, Sensitivity and Specificity. Emerg (Tehran). 2015;3:48-9.

20. Mueller WH, Martorell R. Reliability and accuracy of measurement. In: Lohman TG, Roche AF, Martorell R, editors. Anthropometric standardization reference manual. Champaign: Human Kinetics Books; 1991. pp. 83-6.

21. Norton K, Olds T, editors. Antropometrica. Argentina: Biosystem; 2000.

22. Sullivan JC, Tavassoli T, Armstrong K, Baron-Cohen S, Humphrey A. Reliability of self, parental, and researcher measurements of head circumference. Mol Autism. 2014;5:2. 